Lingüística

Vol. 33-2, diciembre 2017: 125-147

ISSN 2079-312X en línea

DOI: $10.5935 / 2079-312 X .20170020$

\title{
GRAMATICALIZACIÓN COMPARTIDA O HEREDADA VS. INDUCIDA POR EL CONTACTO: CAMBIO LINGÜÍSTICO EN LENGUAS YUTO-AZTECAS DEL NOROESTE DE MÉXICO*
}

\author{
SHARED OR INHERITED GRAMATICALIZATION VS. CONTACT-INDUCED: \\ LINGUISTIC CHANGE IN UTO-AZTECAN LANGUAGES FROM NORTHWESTERN \\ MEXICO
}

\author{
Zarina Estrada Fernández \\ Universidad de Sonora \\ zarinaef@gmail.com
}

\section{Resumen}

El objetivo de este trabajo es establecer la naturaleza precisa de las rutas de gramaticalización que afectan tres tipos de construcciones en lenguas yutoaztecas del noroeste de México: el caso de un clasificador de posesión animal, los marcadores de verbos de voz media y las estrategias de relativización. El análisis es crucial para mostrar que los fenómenos bajo estudio se circunscriben dentro del llamado cambio lingüístico, concretamente de la gramaticalización, en donde se observa procesos de cambio de elementos léxicos a gramaticales y de otros ya gramaticalizados a más gramaticalizados. Además, el análisis destaca que la tipología intragenética es relevante para mostrar casos de gramaticalización inherente, inducida por el contacto y la de tipo areal. El análisis también enfatiza que en los procesos de gramaticalización la extensión semántica y la erosión fonológica no son siempre procesos de gramaticalización relevantes, y que el más importante es la decategorialización.

Palabras clave: gramaticalización heredada; gramaticalización inducida por el contacto; cambio lingüístico intragenético; perspectiva areal; lenguas yutoaztecas

The aim of this article is to establish the precise nature of the grammaticalization pathways that affect three different type of constructions: a classifier of domestic animal possession, markers of middle verbs and relativization strategies on the verb. The analysis is crucial to show that the phenomena under consideration fall within study of linguistic change, in particular of grammaticalization, where either, lexical elements change into

\footnotetext{
* Este trabajo ha sido posible gracias a las aportaciones recibidas a través del proyecto de investigación Tipología intragenética y diversidad lingüística en lenguas de la Tarahumara (CB2013: 220328) financiado por el Consejo Nacional de Ciencia y Tecnología.
} 
grammatical ones, or previously grammatical elements grammaticalizes more. The analysis also shows that an intragenetic typology perspective is relevant to deal with cases of inherent, contact-induced and areal grammaticalization. The analysis also emphasizes that within the process of grammaticalization, semantic extension or phonological reduction are not always relevant processes for grammaticalization and that the most important is decategorialization.

Key words: inherited grammaticalization; contact-induced grammaticalization; intragenetic change; areal perspective; Uto-Aztecan languages

Recibido: $12 / 10 / 2016$

Aceptado: 20/12/2016

\section{Introducción}

El análisis de la gramaticalización de fenómenos de cambio lingüístico requiere de la aplicación del método comparativo al centrarse en lenguas de una misma familia, es decir, desde una visión intragenética. Sin embargo, también es indispensable asumir una perspectiva tipológica intergenética que permita ampliar la observación de los fenómenos de cambio, considerando lenguas de diversas familias lingüísticas, acudiendo en primera instancia a aquellas que se hablan o hablaban en los límites geográficos del primer grupo de lenguas. Esto último repercute en observaciones de cambio inducido por el contacto. Esta visión se asume en este trabajo al abordar la gramaticalización de (i) un clasificador de posesión animal, (ii) la codificación de los marcadores de voz media y (iii) la gramaticalización de un relativizador, cuyo origen se encuentra en un elemento demostrativo.

El estudio de los tres distintos aspectos gramaticales, mencionados anteriormente, en un grupo de lenguas yuto-aztecas habladas en el noroeste de México permite mostrar los procesos de cambio que las diferencian entre sí y su posible explicación como resultado de procesos de gramaticalización heredados. Sin embargo, también se destaca que para la explicación de otro de estos aspectos gramaticales se requiere de la ampliación de la dimensión de lenguas analizadas. Esta cobertura de mayor dimensión apuntala a la conveniencia de considerar lo que sucede en lenguas de otras familias circunscritas a espacios geográficos compartidos de manera inmediata, como es el caso de la lengua seri (aislada), así como de otras lenguas que abren la posibilidad de dimensionar posibles relaciones areales, lo que es posible al atender a la lengua coahuilteca (hokana).

Las lenguas en las que se centra esta contribución pertenecen a la familia yuto-azteca (yuto-nahua); la región donde se hablan es en el noroeste de México, en los estados de Sonora y Chihuahua. Tres de las lenguas pertenecen a la rama taracahita: yaqui, guarijío (warihío) y tarahumara, y cuatro a la 
rama tepimana: tohono o'odham (en México conocida como o'otam), pima bajo, tepehuano del norte y tepehuano del sur ${ }^{1}$.

\section{Nociones relevantes}

La gramaticalización ha sido definida como el proceso mediante el cual, un elemento léxico o gramatical desarrolla o adquiere un nuevo estatus gramatical. La noción de gramaticalización se ha utilizado desde los inicios del siglo $X X^{2}$, aunque a partir de Heine, Claudi y Hünnemeyer (1991), Lehmann (2002), Heine y Kuteva (2007), otros autores prefieren hablar de gramaticalización como un concepto útil para explicar el cambio o evolución de las lenguas (Wiemer 2014), y otros distinguen entre gramaticalización primaria y secundaria (Givón 1991, Bisang 2015).

En general, la gramaticalización permite explicar procesos de cambio lingüístico paulatino o gradual que afectan a un determinado elemento lingüístico. Dicha visión muestra cómo elementos particulares cambian su función dando lugar a otros de mayor sistematicidad o regularidad, al grado de conformar paradigmas morfológicos fuertemente sistematizados (Lehmann 2002), dígase con mayor especificidad gramatical. Sin embargo, como lo ha propuesto Givón (1991), no todos los procesos de cambio o evolución arriban necesariamente a formas morfológicas de alta sistematización (primary grammaticalization), sino que también pueden mostrar los cambios de una forma léxica con una función gramatical determinada (sintacticización), a otras con mayor especificidad funcional, dígase cambio de elementos menos gramaticalizados a más gramaticalizados o funcionales. En este proceso, la pérdida de significado y la erosión fonológica no son siempre los más relevantes sino la decategorización, es decir, el hecho de que el elemento bajo consideración esté sujeto a un cambio de categoría (Heine y Kuteva 2007, Shibatani 2007).

A partir de esta visión, todos los procesos de cambio de una lengua pueden explicarse mediante la noción de gramaticalización siempre que involucren un funcionamiento gramatical. Algunos de estos cambios también se observan en los procesos de cambio inducidos por el contacto. De ahí, que el presente estudio impulsa un modelo que da cuenta de:

A. procesos de cambio que claramente enfatizan la gramaticalización que emerge del análisis comparativo intragenético de un determinado elemento lingüístico, dígase gramaticalización heredada o compartida, cuya cobertura se centra en lenguas de la misma familia, y

\footnotetext{
${ }^{1}$ El tepehuano del sur o sureste no es considerado sistemáticamente en mis comparaciones debido a que los hablantes de esta lengua habitan regiones que se encuentran en los límites de dos zonas culturales distintas: Mesoamérica y Aridoamérica, y por ello, la lengua es susceptible de mostrar comportamientos lingüísticos no necesariamente homogéneos con aquellos de las lenguas en las que se centra esta contribución.

2 Meillet, (1912), Kuryłowicz, (1965), Givón, (1971).
} 
B. gramaticalización que emerge de la interacción entre hablantes que se encuentran en contacto por razones políticas y económicas relacionadas con algún evento socio-político complejo como fue la colonización y que se observa en contextos de lenguas en contacto directo (como es el caso del contacto entre las lenguas indígenas y el español), y gramaticalización emanada del contacto desde una visión areal, que también podría implicar la intervención de lenguas intermediarias, lo que exige ampliar el foco de la geografía lingüística limítrofe de las lenguas en las que nos centramos, para dar finalmente una explicación con mayor certeza científica.

Por lo tanto, el modelo de análisis propuesto atiende los cambios lingüísticos que emergen en lenguas de una misma familia, considerando los cambios internos dentro de la especificidad tipológica que las caracterizan, así como aquellos inducidos por el contacto y su asociación con patrones o procesos de gramaticalización que se han observado en distintas lenguas del mundo. La última perspectiva difiere de la que atiende a los cambios intragenéticos en que se dimensiona el nivel translingüístico.

\section{Lenguas yuto-aztecas del noroeste de México}

Lenguas consideradas: tarahumara, guarijío, tepehuano del norte ypima bajo (con algunas referencias altohono o'odham -antes pápago-), yaqui, tepehuano del sur, e incluso, a lenguas muertas, entre ellas névome y ópata.

Asentamientos: Los límites geográficos donde se hablan estas lenguas se reconocen como noroeste de México; la región donde se asientan los hablantes de cuatro de estas lenguas como Sierra Tarahumarao Sierra Madre Occidental.

Etiqueta lingüística: Un sistema bien organizado del uso retórico de la lengua ha sido observado en los sermones (nawésaris) que los siriames o gobernadores tradicionales del pueblo rarámuri o tarahumara expresan en diversas ocasiones y cuya trascendencia incide en los lazos de su identidad étnica. Entre los yaquis, en cambio, el discurso formal tradicional se utiliza como una herramienta en la "lucha contra el yori", es decir, frente a las personas que no pertenecen al grupo.

Multilingüismo: Notorio entre los pima bajo, que normalmente se supeditan a la hegemonía que tienen los tarahumara y yaqui.

Influencias culturales: a. Fuertemente independiente. Los hombres tarahumarasaún conservan formas de vestir ancestrales. Entre los otros pueblos, solo la mujer tepehuana y yaqui conservan un vestuario que puede ser considerado tradicional; b. Fuertemente dependiente. Los pima bajo y guarijío que en general ya no conservan formas tradicionales en sus vestiduras. 


\section{Posibles direcciones de influencia ${ }^{3}$ :}

Fuerte influencia: tarahumara sobre los pima bajo

Influencia débil: yaqui sobre los guarijío

Influencia incierta: tarahumara sobre los guarijío

Influencia probable: tepehuanos del norte sobre algunos dialectos del tarahumara (zona de Turuachi, Chihuahua)

Tabla 1. Factores sociolingüísticos de las lenguas yuto-aztecas del noroeste de México

\section{Patrones de gramaticalización en lenguas yuto-aztecas del noroeste de México}

En esta sección, nos centramos en el análisis de tres fenómenos de cambio lingüístico atendiendo a las distintas opciones de gramaticalización, estos son: (1) un clasificador de posesión animal, (2) los marcadores de eventos de voz media y (3) los recursos verbales de marcación de relativización.

\subsection{Lo manifiestamente heredado: el clasificador de posesión animal}

Con base en observaciones preliminares provenientes del guarijío, Miller (1980) propone una forma nominal *puku, a la que le asigna el valor semántico de 'mascota', 'esclavo' o 'persona adoptada', como presente en la protolengua yuto-azteca (PYA). La ocurrencia de cognados relacionados con esa forma reconstruida en las lenguas yuto-aztecas que se abordan en esta contribución, permite mostrar un proceso de gramaticalización claramente heredado (inherited grammaticalization). Ejemplos de lenguas taracahitas, tarahumara, guarijío y yaqui, apoyan la argumentación de que el origen de este clasificador se debe ubicar en un verbo de pertenencia o posesión. En (1ab) se observa tanto el uso verbal como el nominal en datos históricos de tarahumara provenientes de un compendio gramatical colonial (Guadalaxara 1683), así como de un manuscrito gramatical escrito en latín de Steffel (1799); en (1c) el uso nominal en la obra reciente de Brambila (1953). El término solo muestra la sonorización de la consonante /p/ propuesta por Miller para la forma reconstruida:

1. Tarahumara buku:

a. bucúrú bucurúqui 'tener/tomar a alguien como esclavo', 'tener/tomar a una bestia'(Guadalaxara, 1683)

\footnotetext{
${ }^{3}$ Las hipótesis de cambio se plantean con base en los asentamientos y en el tipo de tierras en los que estos pueblos se asientan.
} 
b. bucu nombre genérico para 'bestia', 'animal'; Pedro bucúla 'el caballode Pedro'; Ihé bucú 'este rebaño' (Steffel, 1799) $\begin{array}{cllllll}\text { c. we } & \text { risió } & \text { rútzane } & \text { kemu } & \text { bukú } & \text { Lirio } & \text { asegá } \\ \text { INT } & \text { cansada } & \text { cabalgar } & \text { 3sG.POS } & \text { caballo } & \text { Lirio } & \text { dice }^{4}\end{array}$

'Lirio dice que ella está cansada de cabalgar en su caballo.' (Brambila, 1953: 385)

En los datos de yaqui, según se observa en (2), el cognado del clasificador conserva tanto su uso verbal como nominal:

2. Yaqui buke:
a. Joan wakas-im buke.
Juan vaca-PL pastorear.PRS
'Juan pastorea vacas.'
b. U buki bwia-po yeewe.
DET.SG.SUJ criatura tierra-LOC jugar.PRS
'La criatura juega en la tierra.'

En guarijío, que junto con el tarahumara y yaqui forman parte de la rama taracahita, también se observa al cognado mencionado por Miller (1980), aunque en esta lengua se atestigua solamente el uso no obligatorio del clasificador de posesión de animal doméstico:

3. Guarijío puhku:
$\begin{array}{lll}\text { a. no'ó } & \text { puhku-wá } & \text { čuhčúri } \\ \text { 1SG.NSUJ } & \text { CLAS-POS } & \text { perro }\end{array}$
'mi perro'
b. no'ó čuhčúri-wa
1SG.NSUJ perro-POS
'mi perro' (Félix 2007: 17)

En lenguas yuto-aztecas de la rama tepimana: pima bajo, tepehuano del norte $^{5}$, se atestigua un clasificador de diferente origen léxico. Véase en (4) un ejemplo de pima bajo y en (5) de la lengua tepehuano del norte:

4. Pima Bajo soig:

\begin{tabular}{|c|c|c|}
\hline $\begin{array}{l}\text { huaan } \\
\text { Juan }\end{array}$ & $\begin{array}{l}\text { gogos } \\
\text { perro }\end{array}$ & $\begin{array}{l}\text { in } \\
\text { 1SG.NSUJ }\end{array}$ \\
\hline
\end{tabular}

'Juan mató a mi perro (mascota).'

5. Tepehuano del norte soiga:

$\begin{array}{llll}\text { gin } & \text { soiga } & \text { gogooši } & \text { muu. } \\ \text { 1SG.POS CLAS } & \text { perro } & \text { morir.PFV } \\ \text { 'Mi perro murió.' } & & \end{array}$

Al contrario de lo que sucede en las dos lenguas tepimanas recién mencionadas, en tepehuano del sur, o sureste, lengua hablada en el estado de Durango en los límites del noroeste mexicano e inicio del área de Mesoamérica, sí se ha conseguido documentar tanto al clasificador de posesión animal como

\footnotetext{
${ }^{4}$ Las glosas son resultado de mi análisis.

${ }^{5}$ Para el o'otam o Tohono O'odham -en inglés- se hace necesaria más investigación.

6 En las lenguas tepimanas el fonema /s/ tiende a palatalizarse como [š] en presencia, no necesariamente inmediata, de una vocal alta /i/.
} 
a la forma verbal a partir de la cual se origina el clasificador; véase en (6a) la forma del clasificador y en (6b) la del verbo, lo que apuntala la ruta de gramaticalización so'm 'cuidar.DUR>'šoi? 'clasificador de posesión de animal doméstico':

6. Tepehuano del sureste: 'šoi? 'clasificador de posesión animal' (Willett, 1991: 16)

a. Hum šoi?

2SG.POS CLAS

'Tu animal domesticado.'

Tepehuano del sur (García Salido, c.p.) ${ }^{7}$

b. bhai' ñich ja'p ba t+bh+ich gu tatoxkolh tu so'm dhidha' iñ

bhai' ñich ja'p ba-t+bh+ich gu ta-toxkolh,tu-so'm-dhi-dha'-iñ

DIR 1SG.SUJ DIR COMP-pasar ART RED-puercos DUR-cuidar-APL-CONT-1SG.SUJ

'por ahí pasé los puercos, yo los cuidaba...' (Texto_092010_TSC_GGS_nar +lhich ka', 02: 34)

Los datos presentados hasta aquí permiten sostener la gramaticalización de un clasificador de posesión de animal doméstico a partir de un elemento verbal (verbo de pertenencia > clasificador de posesión animal). La presencia de nombres que aluden a 'criatura', 'esclavo' o 'animal doméstico' permiten ampliar dicha ruta agregando una etapa intermedia que seguramente dio lugar al carácter nominal del elemento, lo que implica la evolución del elemento verbal a un nominal de mayor especificidad semántica y finalmente la de un segundo cambio de categoría que genera al clasificador nominal (verbo de pertenencia $>$ nombre que alude a persona dependiente -criatura o esclavo0 , animal doméstico > clasificador de pertenencia de animal doméstico).

El que lenguas de la rama taracahita muestren un cognado afín a la forma propuesta por Miller (1980) y que las tepimanas, en cambio, expresen una noción similar, pero mediante otra forma léxica, tiene dos posibles interpretaciones: la selección de dos elementos distintos que compartían significados similares, o bien, que el origen de la diferente forma léxica se encuentre relacionada con factores relativos a la historia de la separación de estas dos ramas de la familia yuto-azteca. Pese a ello, la ruta de gramaticalización del elemento verbal a clasificador nominal con un estado intermedio que ubica a un nominal es obvia. Se trata de un fenómeno de cambio compartido o heredado, aunque la diferencia del recurso léxico podría apuntalar a influencias del contacto lingüístico si se pudiera determinar que durante el proceso de migración social de norte a sur, los hablantes de lenguas tepimanas tuvieron una época de contacto con lenguas del suroeste del área de California de donde tomaron la forma léxica de su clasificador.

\subsection{Marcadores de voz media: cambio inducido por el contacto}

En su obra sobre verbos medios, reflexivos y voz media, Klaiman (1992: 37-38) señala que aunque las construcciones medias son formalmente reflexivas, su semántica no es tal. En su estudio indica que hay tres nociones relacionadas con la media; dos relacionadas con la dediátesis, y la tercera

\footnotetext{
7 Agradezco a Gabriela García Salido (comunicación personal, c.p.) por haber compartido conmigo algunos datos de la base extensa que ha construido durante su investigación doctoral (García Salido, 2014) y posteriormente a ella.
} 
como propiedad flexiva. La primera noción considera a las construcciones medias como aquellas en las que el rol semántico del sujeto es de paciente (patient-like); la segunda ubica a estas construcciones como reflexivas; y la última como una oposición flexiva que se localiza en la conjugación verbal (la tradicional voz media del griego), lo cual es retomado por Manney, (2000: 15) al ubicar al griego antiguo y moderno como lenguas donde la voz media ${ }^{8}$ es parte de la marcación flexiva del verbo.

Otros autores, señalan que solo cierto tipo de verbos deben considerarse como específicos de voz media y por ello pueden comportarse como transitivos o intransitivos (Mel'čuk 1993: 21) ${ }^{9}$. Esta postura contrasta con el análisis desarrollado por Kemmer (1993, 1994), al proponer que son varias las clases verbales que muestran un comportamiento característico de la voz media (conjunto de verbos que agrupa en el dominio semántico de la media). Los valores semánticos de los verbos que conforman ese dominio, según esta autora, se incluyen en la Tabla 2:

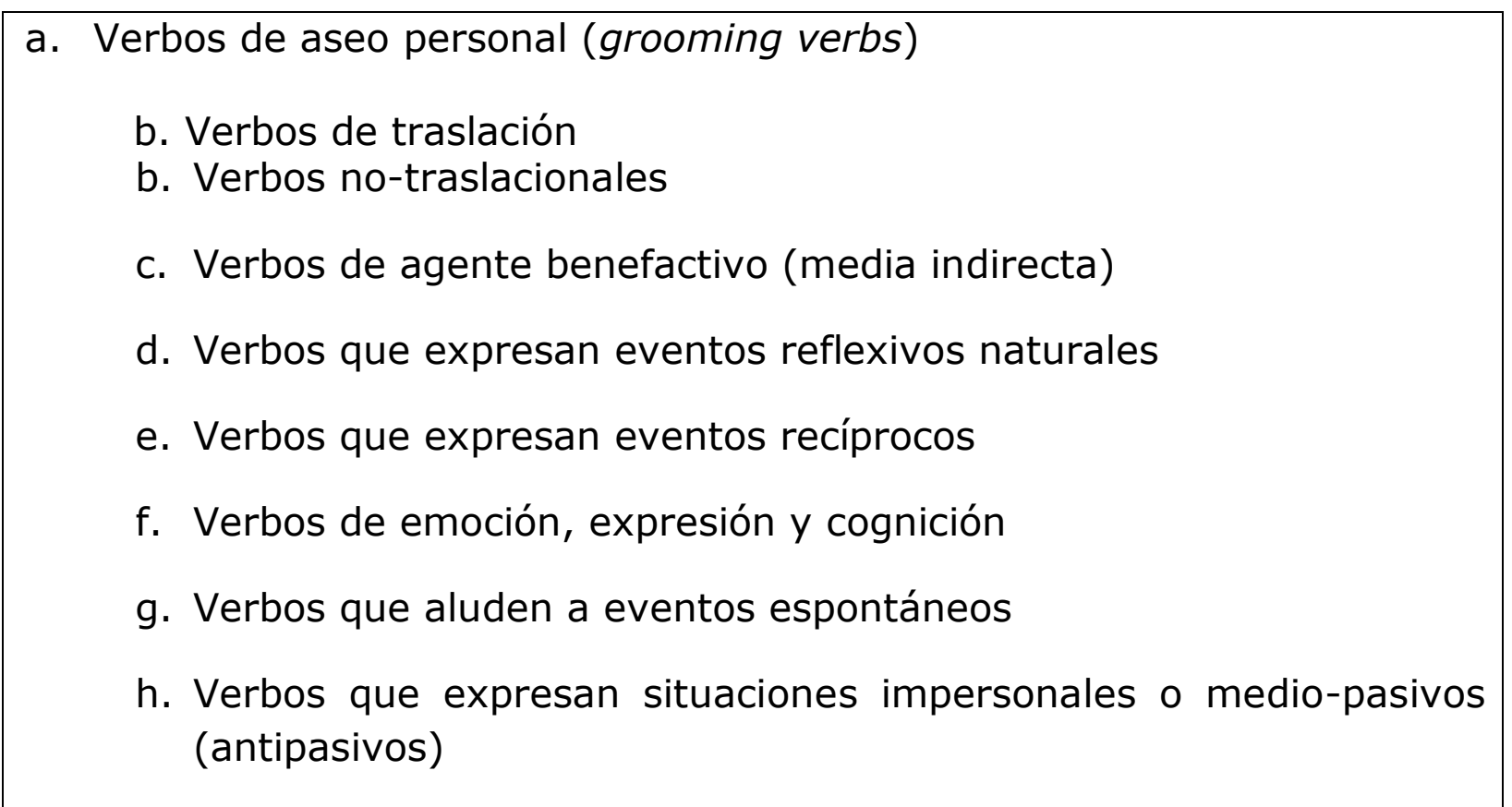

Tabla 2. Verbos del dominio de la media según

El estudio de las estrategias de codificación de las situaciones que se encuentran en el dominio semántico de la media en las lenguas yuto-aztecas del noroeste de México, permite proponer tres distintos escenarios:

Lenguas no-marcadas: Guarijío (warihío) y tarahumara. Estas lenguas gramaticalizan las situaciones medias como verbos intransitivos (excepto los de las clases e y $\mathrm{f}$ de la Tabla 2). En tarahumara un reducido número de verbos de aseo personal se comporta como transitivos.

\footnotetext{
8 Otros nombres usados para referirse a estas construcciones son: media/pasiva, cuasireflexiva, pseudo-reflexiva, neutral, construcción con sujeto paciente y verbo deponente. A los recíprocos Mel'čuk (1993: 22) los denomina también de carácter mutuo (reciprocal or mutual carácter of the action).

${ }^{9}$ Haspelmath (1993: 92) Ilama lábiles a estos verbos.
} 
Lenguas marcadas: Yaqui. Esta lengua gramaticaliza estos eventos regularmente como intransitivos y solo un reducido número, generalmente de aseo personal (clase a en la Tabla 2), como reflexivos.

Lenguas marcadas: Existen dos grupos: (i) tepehuano del norte, donde la mayoría de los verbos que expresan situaciones medias se comportan como reflexivos, aunque algunos son intransitivos y (ii) pima bajo y tepehuano del sur, donde la mayor parte de los verbos en la Tabla 2, se gramaticalizan como construcciones medias, solamente dos clases verbales, e-f, en Tabla 2, se mantienen como reflexivos.

En la Tabla 3 se presenta el resumen de los comportamientos que los verbos del dominio de la media mostraron en las distintas lenguas analizadas (Estrada 2005, Estrada 2006, Estrada y Félix Armendáriz 2010, García y Estrada 2009):

\begin{tabular}{|c|c|c|c|c|c|c|}
\hline Verbos & $\mathrm{Gu}$ & Tar & Yaq & TN & PB & TS \\
\hline Verbos de aseo personal & Intr & Verbos lábiles & Intr/Rflx & Rflx & Intr/Rflx/M ${ }^{10}$ & M \\
\hline Verbos de traslación & Intr & Intr & Intr & Intr & Intr $^{11} / \mathrm{M}$ & Intr/M \\
\hline Verbos de no traslación & Intr & Intr & Intr/Rflx & Intr/Rflx & $\mathrm{M}$ & $\mathrm{M}$ \\
\hline $\begin{array}{l}\text { Verbos de cambio de } \\
\text { postura corporal }\end{array}$ & Intr & $\operatorname{Intr} /(\mathrm{Rflx})^{12}$ & Intr & Intr/Rflx & $M$ & $M$ \\
\hline $\begin{array}{l}\text { Verbos que expresan } \\
\text { eventos naturalmente } \\
\text { reflexivos }\end{array}$ & Rflx & Rflx & Rflx & Rflx & Rflx & Rflx \\
\hline Recíprocos & Rflx & Rflx & Rflx & Rflx & Rflx & Rflx \\
\hline $\begin{array}{l}\text { Verbos de emoción, } \\
\text { expresión y cognición }\end{array}$ & Intr & Intr & Intr & Intr & $M$ & $M$ \\
\hline Eventos espontáneos & Intr & Intr & Intr & Intr & $M$ & M \\
\hline $\begin{array}{l}\text { Pasivas impersonales o } \\
\text { medio pasivas }\end{array}$ & Intr & Intr & Intr & Intr & M & M \\
\hline
\end{tabular}

Tabla3. Eventos medios en lenguas yuto-aztecas del noroeste de México

En (7) y (8) se ilustran verbos de la clase (c), en guarijío y clase (a), en tarahumara, de acuerdo a la Tabla 2 . Nótese que estas expresiones ocurren como intransitivas, es decir, solamente se codifica un argumento en el verbo:

7. Guarijio/Warihio (construcción intransitiva)

kuri'-ru=ne.

voltear.cara-PFV.EVI $=1$ SG.SUJ

'Giré la cara.'

8. Tarahumara (construcción intransitiva/transitiva, verbos lábiles)
a. nejee
rapako
uba-ri.
1SG.SUJ
ayer
bañar-PFV
'Yo (me) bañé ayer.'

\footnotetext{
${ }^{10}$ Verbos transferidos del español al pima bajo se codifican como eventos medio.

11 Contextos pragmático-culturales hacen posible la codificación media de ciertos verbos de movimiento con traslación.

12 Raras.
} 
$\begin{array}{llll}\text { b. echi towi rapako ne } & \text { uba-ri. } \\ \text { DET niño ayer } & \text { 1SG.SUJ } & \text { bañar-PFV } \\ \text { 'Yo bañé al niño ayer.' } & & \end{array}$

De manera similar, en yaqui, la mayor parte de los verbos del dominio de la media ocurren como intransitivos, excepto los que expresan eventos de cuidado o aseo personal -clase (a) en la Tabla 2- que se gramaticalizan como reflexivos. En (9a) se destaca el argumento único del verbo po'okte 'inclinar' que está codificado mediante un pronombre nominativo ne '1SG.NOM', mientras que en (9b) ocurren dos argumentos, u jamut 'la mujer' y el pronombre reflexivo emo '3RFLX', que identifica a la misma entidad pero en su rol de paciente porque sobre ella recae la acción del peinarse:

9. Yaqui

a. ne po'okte-k.

1SG.NOM inclinar-PFV

'Me incliné.'

b. $u$ jamut emo chike.

DET.SUJ mujer 3RFLX peinar.PRS

'La mujer se peina (a sí misma).'

En contraste con las lenguas de la rama taracahita, las pertenecientes a la rama tepimana resaltan por su tendencia a gramaticalizar de forma muy idiosincrásica las construcciones que contienen verbos del dominio de la voz media. Esto se observa claramente en tepehuano del norte: en (10a-b), se ilustran verbos de movimiento no traslacional (clase c en la Tabla 2) codificados como reflexivos, lo que se observa es la presencia del clítico, $=n i$ '1SG.SUJ' para referirse al sujeto y el pronombre no-sujeto, giñ- '1SG.NSUJ', que denota alreflexivo o paciente. En (10c) se ilustra un evento de aseo personal, donde el pronombre independiente enfatiza al agente y el clítico giñ- '1SG.NSUJ', al patrón reflexivo:

10. Tepehuano del norte
a. giñ nasaa $=n i$
1SG.NSUJ inclinar.PASD $=1 \mathrm{SG} . \mathrm{SU}$
'Yo me incliné.'
b. $g \dot{i} \tilde{n} \quad$ to-toona-kidj kikitva $=n i$
'me hinqué.'
1SG.NSUJ RDP-rodilla-POSP parar.PASD $=1 \mathrm{SG} . \mathrm{SU}$
c. aani vakoi giñ buibasu
1SG.SUJ lavar.PAS 1SG.NSUJ cara
'yo me lavé la cara.'

En pima bajo, en cambio, un pronombre de tercera persona no-específica, $a-$, ha sido gramaticalizado como marcador de media para todas las personas del paradigma. La gramaticalización de dicho pronombre se hace evidente, ya que impide que exista una relación de correferencia entre el pronombre que codifica al participante paciente y el sujeto-agente. El resultado de esto determina que se presenten tres tipos de comportamiento: (a) el intransitivo prototípico, (b) el reflexivo y (c) el que consideramos de voz media (Estrada 
2005). En (11) se ilustran las tres posibilidades: en (11a), el uso prototípicamente intransitivo de un verbo de movimiento; en (11b), una construcción reflexiva con un verbo de aseo o limpieza personal. Asimismo, los ejemplos en (11c-d) muestran el comportamiento en un verbo de movimiento no traslacional, (11c), y de movimiento simple, en (11d), en un evento de movimiento simple en perfectivo. El contraste de estos tres comportamientos sintácticos es nítido al observar los patrones de uso de los pronombres. En los cuatro ejemplos, el pronombre reducido de sujeto aan '1SG-SUJ' codifica al argumento agente del verbo, mientras que en (11b) el pronombre prefijado in'1SG-NSUJ', codifica al reflexivo paciente y en (11c-d), el elemento prefijado al verbo, $a-$, históricamente un pronombre de tercera persona no-específico, indica que se ha gramaticalizado como marcador de media, por lo que no tiene ya la posibilidad de codificar correferencia entre los argumentos.

11. Pima bajo
a. aan hima
1SG.SUJ ir.IMPF
'Yo voy.' (yo me voy)
$\begin{array}{ll}\text { b. aan in-vak-im. } \\ \text { 1SG.SUJ } & \text { 1SG.NSUJ-bañar-CONT }\end{array}$
'Yo me estoy bañando.' (a mí mismo)
c. aan a-tiiv-va.
1SG.SUJ MED-inclinar-COMP
'Yo me incliné.'
d. aan a-him-va.
1SG.SUJ MED-ir-COMP
'Yo me fui.' (ya me fui)

El tepehuano del sur también se comporta como el pima bajo, solo que su marcador de media se ha gramaticalizado a partir de un pronombre diferente (García y Estrada 2009). En general, el tepehuano del sur también codifica los eventos del dominio de la media de manera idiosincrásica porque tiende a marcar a los de movimiento simple como intransitivos, y a los de movimiento no traslacional y de aseo o cuidado personal como reflexivos. En (12a) se ilustra el uso reflexivo prototípico, donde se muestra la correferencia entre el pronombre reflexivo con el de sujeto. ${ }^{13}$ En cambio, (12b) ilustra al pronombre de segunda persona singular no-sujeto, jum =, gramaticalizado como marcador de media, ya que el pronombre se ha extendido a la segunda y tercera persona del singular o plural. En (12c), se observa que el cambio no afecta a la primera persona que aún continúa mostrando un comportamiento reflexivo. La decategorización del pronombre se demuestra no solo por la extensión de su significado, sino por el hecho de haberse generalizado como marcador de media.

12. Tepehuano del sur
a. ap jum=kok-tu-lh.
2SG 2SG.RFLX=golpear-CAUS-CAUS
'Tú te golpeaste.'

${ }^{13}$ Los ejemplos han sido tomados de García (2007) y García y Estrada (2009). 
b. guñi jum=to'm.

3SG MED=estirarse.PASD

'Ella se estiró.'

c. añ jiñn=cho'm.

1SG 1SG.RFLX=estirarse.PASD

'Yo me estiré.'

En la tabla 3 se destacan por medio de números romanos (I, II, y III) los tipos de gramaticalización que se observan en los verbos del dominio de la voz media (gramaticalización intransitiva, media y reflexiva) en las distintas lenguas estudiadas (Estrada y Félix Armendáriz 2010). Asimismo, se caracteriza el tipo de codificación que estas lenguas ofrecen, de manera general, para las expresiones que se encuentran en los distintos dominios semánticos de la voz media.

\begin{tabular}{|llll|}
\hline I & II & III & \\
& Intransitiva & Media & Reflexiva \\
\hline Guarijio & $\mathrm{X}$ & -- & $\mathrm{X}$ \\
Tarahumara & $\mathrm{X}$ & -- & $\mathrm{X}$ \\
Yaqui & $\mathrm{X}$ & -- & $\mathrm{X}$ \\
Tepeh. N. & $\mathrm{X}$ & -- & $\mathrm{X}$ \\
Pima Bajo & $\mathrm{X}$ & $\mathrm{X}$ & $\mathrm{X}$ \\
Tepeh. S. & -- & $\mathrm{X}$ & $\mathrm{X}$ \\
\hline
\end{tabular}

Tabla4. Etapas de gramaticalización de lenguas yuto-aztecas del noroeste de México

El escenario lingüístico que involucra a las distintas construcciones ubicadas dentro del ámbito de la voz media, descritas en este apartado, es posiblemente resultado de las siguientes rutas de cambio o gramaticalización:

A. Una ruta es aquella donde las construcciones responden a una gramaticalización heredada. Nos referimos a un comportamiento característico donde la mayor parte de estos verbos se comportan como eventos intransitivos. Este comportamiento tiende a emerger en casi todas las lenguas: guarijío, yaqui, tarahumara, algunas construcciones en pima bajo y tepehuano del norte.

B. Otra ruta corresponde a las construcciones medias que muy posiblemente se hayan generado por contacto con el español. Esto se ha mostrado en pima bajo y tepehuano del sur.

Sin embargo, no es posible aún proponer un análisis contundente; principalmente por la falta de documentación histórica que permita confirmar esto. En las lenguas tepimanas existen dos propiedades tipológicas relevantes que pudieron haber influido en la codificación de las construcciones medias: (a) la primera es el hecho de que las lenguas tepimanas carecen de morfología pasiva, y (b) la segunda es que las lenguas tepimanas carecen de un pronombre de tercera persona singular. De esta manera, la estrategia del pima 
bajo fue la de utilizar un pronombre de tercera persona no-específico como marcador de las construcciones medias, y la de tepehuano del sur fue extender el pronombre de segunda persona para la segunda y tercera persona en singular y plural. Este comportamiento, el que elementos con cierto grado de ambigüedad sean reclutados para codificar una nueva función gramatical, se ha considerado factor importante en los procesos de cambio lingüístico.

Finalmente hay que observar que a pesar de que el tepehuano del norte no gramaticaliza los eventos de voz media como construcciones medias, sí los ha resuelto como construcciones reflexivas, algo que seguramente también es resultado del contacto con el español.

\subsection{Construcciones relativas y procesos de cambio}

Las construcciones relativas, por su semántica, han sido vistas como modificadores nominales que generalmente forman parte de una frase nominal (Givón 2001: 175). Heine y Kuteva (2002: 113-115, 174, 251) han propuesto que la gramaticalización de los relativizadores tiene principalmente su origen en el cambio que afecta a elementos léxicos o funcionales, (i-iii), aunque otros autores añaden los que se enlistan en (iv-v):

Procesos de gramaticalización de las construcciones relativas: ${ }^{14}$

i. Demostrativo > Relativo (en Canela-Krahô ${ }^{15}$, Popjes y Popjes 1986: 171)

ii. Locativo (Here) $>$ Relativo (en Buang ${ }^{16}$, Sankoff 1979: 35-6)

iii. Interrogativo (W-word)> Relativo (en Baka ${ }^{17}$ (là), Heine y Kuteva 2002: 251)

iv. Elemento retórico (tag-particle)> Relativo (en Tamil, Herring 1991: 275$276)^{18}$

v. Especificador> Relativo (complementante) (Gelderen 2004: 81ff)

En las lenguas yuto-aztecas del noroeste de México, la gramaticalización de los elementos relativizadores muestra, en general, tres distintos orígenes: (a) sufijos nominalizadores o deverbales, (b) subordinantes y (c) gramaticalización de un nominalizador complejo que emerge de un sufijo aspectual estativo que se fusiona con un demostrativo.

\subsubsection{Gramaticalización de cláusulas relativas por medio de sufijos nominalizadores}

Bascom (1982), observa que las construcciones relativas no-finitas de tepehuano del norte se obtienen a través de un sufijo nominalizador -kame/kami (glosado por el autor como participio, PRTC), como se ilustra en (13): ${ }^{19}$

\footnotetext{
${ }^{14}$ Para las referencias de: Popjes y Popjes 1986, Sankoff 1979, Herring 1991 y Gelderen 2004, consúltese Heine y Kuteva (2002).

15 Macro-Ge, Brasil. ISO 639-3: ram.

${ }^{16}$ Lengua austronesiana de Papúa Nueva Guinea. ISO 639-3: bzh.

17 Lengua nilo-sahara que se habla en Sudán y también en la República Democrática del Congo. ISO 639-3: bdh.

${ }^{18}$ ISO 639-1: ta.

19 Esta estrategia de relativización no se ha identificado en datos documentados en los últimos diez años.
} 
13. Tepehuano del norte
bir-ími-i
†̇mó
gì-vííd’a-kami.
esta.forma-venir-IMPF
uno RFLX-be.rich-NMLZ
'There comes a rich man.' (Bascom, 1982: 378)

Enguarijío de Arechuyvo, Chihuahua (Miller 1996: 179), así como en warihío del río (Félix 2007: 82-83), las construcciones relativas se forman mediante el sufijo derivativo -me, cognado del identificado por Bascom para el tepehuano del norte:

14. Guarijío (Warihío)
a. tihoé
tetewá-ka-me
Hombre ver-PASD-NMLZ soldado
wačó.
'El hombre que vio al soldado.'
b. tihoé karí mete-yáme ko'korépa-re.
hombre casa construir-NMLZ enfermar-PFV
'El hombre que construyó la casa enfermó.'

El sufijo nominalizador -me $\sim$-yame no se observa en relativas de objeto, ya que en estas construcciones el sufijo nominalizador es - $a$.
15. tihoé amó me'eyá-ri-a.
hombre 2SG.NSUJ matar-PFV-NMLZ
'El hombre que tú mataste.'

En tarahumara, el sufijo nominalizador -ame, que alterna con -me, también está restringido a las construcciones relativas de sujeto:

16. Tarahumara
a. rejoi Noroachi bete-ame norina-ri.
hombre Norogachi vivir-NMLZ venir-PFV
'Vino el hombre que vive en Norogachi.'
b. nejee kochií choo-ka-me raraa-ri.
1SG.SUJ perro negro-EST-NMLZ comprar-PFV
'Yo compré el perro que es negro.'

En construcciones relativas de sujeto en yaqui, también se observa un sufijo cognado del nominalizador-(ka)me:

17. Yaqui
U
o'ou
enchi
bicha-ka-me
siika.
DET.SG.NOM
hombre-NOM
2SG.ACU
ver-PFV-NMLZ
ir.SG.PFV

'El hombre que viste se fue.' (Guerrero, 2012: 99)

$\begin{array}{lllll}\text { 18. U-me } & \text { o'ou-im } & \text { em } & \text { bicha-ka-' } u^{20} & \text { saja-k. } \\ \text { DET-PL } & \text { hombre-PL } & \text { 2SG.GEN } & \text { ver-PFV-CLM } & \text { ir.PL-PFV }\end{array}$

'Los hombres que viste, salieron.' (Guerrero, 2012: 99)

\footnotetext{
20 Aunque Guerrero (2012) glosa este elemento como complementante, en realidad debería glosarse como nominalizador ya que se encuentra relacionado con un marcador de caso oblicuo -wi.
} 
Lo mismo sucede en construcciones relativas de lenguas yuto-aztecas ya extintas habladas anteriormente en el noroeste de México, como el ópata y el névome:

19. Ópata
a. Diosa
Dios
2SG.NOM 2SG.GEN
eme nehauere-came-ta.
2SG.GEN hacer.misericordia.PRS-NMLZ-ACU
higuada-te
nererea
corazón-GEN
amar.FUT
'Amarás con todo tu corazón a Dios que es misericordioso.' (Ramírez c.p.) $)^{22}$
b. no
1SG.GEN maizal-GEN
ma oguiqu-ea.
2SG.NOM Ilamar-FUT
'Tú llamarás a los hombres que regarán tu maizal.' (Ramírez c.p.)
vanua-came-ta
urini-ta
hombre-ACU

20. Névome
a. T'-oga
1PL.POS-padre
m'-tu-na
tidamaca-tum'-ami
cielo-LOC-NMLZ
mu-tuguiga.
da-cama
$\mathrm{s}^{\prime}$-cuga
RFLX-glorificado-COND
be-NMLZ PRED-bien
'Nuestro padre, que está en el cielo, sea tu nombre alabado (visto bien).'
(Estrada Fernández, 2012: 138)

Los datos ilustrados de (13) a (20) muestran la fuerte tendencia de las lenguas analizadas a gramaticalizar un relativizador a partir de un sufijo nominalizador. Sin embargo, también otra fuente de gramaticalización de relativizadores la constituyen los subordinantes que se han formado a partir de demostrativos. Esto se mostrará en la siguiente subsección.

\subsubsection{Gramaticalización de cláusulas relativas por medio de elementos subordinantes}

Otra estrategia que se observa en construcciones relativas es la gramaticalización de un subordinante que al combinarse con un demostrativo da origen a un pronombre relativo. La lengua tarahumara muestra esto nítidamente a través de la gramaticalización de mapu, como se ilustra en (21ac). Este nexo subordinante procede del subordinante ma, común en muchas lenguas yuto-aztecas (tohono 'o'odham, tepehuano del sur, náhuatl, sólo para mencionar algunas), ${ }^{23}$ y es el resultado de la combinación de este con un demostrativo pu (Guadalaxara, 1683), hoy ya en desuso. La estrategia de este subordinante complejo se complementa con el uso de un sufijo nominalizador, $-a$, que ocurre en construcciones no-finitas, como en (21a). La estrategia heredada, que se observa en el uso del nominalizador, avanza un paso

${ }^{21}$ La segunda persona posesiva tiene dos formas pronominales alternativas: amo, y eme, la segunda está restringida para los sujetos dependientes.

22 Comunicación personal (c.p.) de Moisés David Ramírez Mendívil que desarrolló una tesis doctoral en esta lengua (Inédita).

${ }^{23}$ En tohono 'o'odham (antes pápago), ma es frecuente al inicio de cláusulas dependientes según se observa en datos de Bahr et al., (1974); en tepehuano del sur García (2014) documenta a un cognado na de este subordinante, en náhuatl, Peralta (2015: 141, ej. 21) también identifica al subordinante ma. 
adelante en el proceso de gramaticalización al sistematizar como obligatorio el uso del conectivo complejo mapu. Datos de la lengua, como se ilustra en (21b) demuestran que la estrategia heredada de la nominalización del verbo tiene rastro evidente de un cambio de categoría, porque en dicho ejemplo permanece aún el sufijo de aspecto perfectivo - ri al lado del nominalizador -a, lo que ha sido visto, bien, como propio del carácter mixto de las nominalizaciones en términos categoriales (Malchukov, 2004), o bien, como la tendencia de carácter universal a la refinitización de la cláusula según Givón (2015, cap. 27). Todo en conjunto permite explicar las tres posibilidades que se observan en (21a-c).

\section{Tarahumara}
a. sebali namuti mapu iyeri ich-a Raramuri... algunas cosas SUB FOC sembrar-NMLZ rarámuri...
'Algunas de esas cosas que el rarámuri siembra...'
b. echi nali wikara-ami ba mapu wili-r-a

DEM LOC cantar-NMLZ DPD SUB traer-PFV-NMLZ

a'li chema la ba...
AF celebración MOD DPD
'El cantor que carga la sonaja durante la celebración...'
c. 'a na rewa-ri echi kochi mapu Juan mea-ri.
AF LOC ver-PFV DEM perro SUB Juan matar-PFV

'Yo vi el perro que Juan mató.'

Para tepehuano del norte, Bascom (1982: 377) también describe cláusulas relativas finitas que hacen uso de unsubordinante, ši $\sim$ iš, que generalmente ocurre como clítico a la izquierda de pronombres demostrativos que hoy funcionan como interrogativos, entre ellos, =máákidi 'quien', en (23a), o =anki 'que', en (23b). ${ }^{24}$

22. Tepehuano del norte
a. káši $=a=n=t \quad$ ií casi= casi $=\mathrm{B}=1 \mathrm{SG}=\mathrm{COMPL}$ beber yo 'Ya tomé la pastilla que él me dio.' go-pastíy
DET-pastilla
iš $=m a ́ a ́ k \dot{t} d \dot{t}$ SUB $=$ INTER
giñ-máá '́gai.
b. $s=$ aid $^{y}$ ika CIT=entonces asustar DET-mujer
ši=anki kóóyi SUB $=$ INTER serpiente 'Entonces la mujer que se convirtió en serpiente se atemorizó.'
1SG.NSUJ-darél
gi-nááto. RFLX-convertirse

La confluencia de un subordinante y un pronombre de sujeto para dar origen a un relativizador, que se usa al inicio de una cláusula, es una ruta de gramaticalización que también se observa en tepehuano del sureste. Véase las construcciones en (24a-b) donde el subordinante na puede ocurrir con el clítico pronominal de segunda persona singular $=p i m$, o de tercera persona $=m$.

23. Tepehuano del sur

a. gu atulh na=pim makia'.

DET atole REL=2SG.SUJ dar.FUT

'El atole que tú darás a ella.' (Text_092010_TMR-GGS_LasTablas3hermanos, 01: 46)

24 Mithun (2012) describe el uso de elementos interrogativos en la codificación de construcciones relativas como un rasgo motivado por el contacto de las lenguas americanas con las europeas. 


$$
\begin{array}{lllcc}
\text { b. mu } & \text { sap } & \text { pix } & \text { ji } & \text { juruñ-amit } \\
\text { DIR } & \text { REP.UI } & \text { MIR } & \text { FOC } & \text { permanecer-3PL.SUJ.PFV } \\
\text { gu } & \text { xi-siogi-'ñ } & & n a=m & \text { tu-juan. } \\
\text { DET } & \text { RDP.PL-hermano-INAL } & \text { REL=3PL.SUJ } & \text { DUR-trabajar }
\end{array}
$$

'Ese de allá, según sus hermanos, que estaba trabajando se quedó (por varios días).' (Text_092010_TMR_GGS_LasTablas3hermanos, 02: 58)

El extremo más gramaticalizado de esta cadena de cambio lingüístico se observa en pima bajo, lengua en la que un sufijo de nominalización estativa, $k a$, se sincretiza con un demostrativo erosionado, higai > -ig, para dar origen a un sufijo relativizador $-k \dot{g}$, no susceptible de ser marcado con persona. En la variedad histórica de esta lengua, névome (Shaul 1986), el relativizador muestra un menor grado de erosión fonética, lo que se observa en la forma ortográfica cugai, en (25):

24. Névome
a. governaro
tu tuanu-cugai
si-bu[h]ogurhida-raga.
gobernador
IT órdenes-REL
INT-obedecer-CAUS
'Las órdenes del gobernador deben obedecerse.'
b. n'-ohana'-cugai s'-amurhida-mut'-api posa pim'an'-t'-io m'-agui. mi-escribir-REL EST-conocer-querer-2SG.SUJ pero NEG-1SG-PFV-FUT tú-decir.PFV 'Tú quieres saber que escribiré, pero no te lo diré.'

En el pima bajo actual, la gramaticalización plena del sufijo relativizador $k \dot{k g}$, muestra otras propiedades de la nominalización de las construcciones relativas, principalmente la expresión de aspecto, en (26a), que valida el carácter mixto. Otro rasgo es la expresión del sujeto nocional de la relativa argumento no-correferente con el nombre núcleo- por medio del pronombre no-sujeto prefijado, am-, como se observa en (26b); este comportamiento equivale al genitivo o acusativo que autores como Givón (2001) sostienen como rasgo característico de las construcciones nominalizadas:

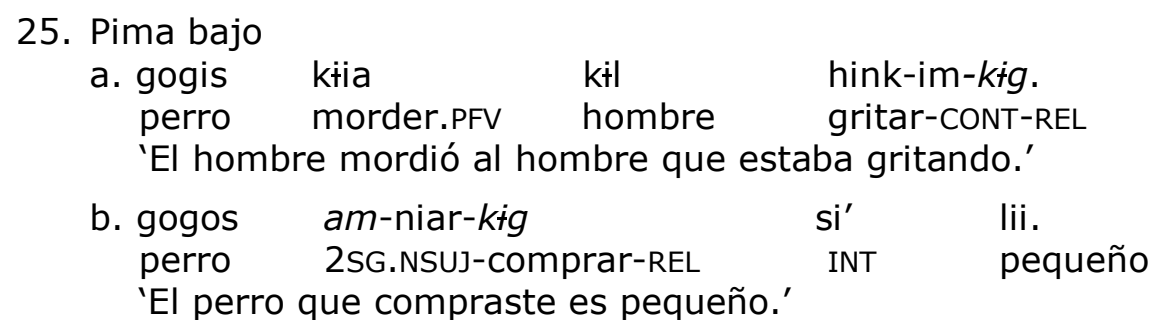

A manera de resumen, en esta sección hemos destacado que la gramaticalización de elementos relativizadores en las lenguas yuto-aztecas abordadas, ha permitido mostrar dos rutas o cadenas como fuerzas que rigen el cambio lingüístico: la morfológica y la sintáctica. La primera se manifiesta a través de la nominalización verbal mediante el uso de sufijos derivativos deverbales; este comportamiento lingüístico es considerado una característica sólida que se asocia a esta familia lingüística (Givón 2016: 271), sobre todo entre las lenguas yuto-aztecas actualmente habladas en el extremo norte del continuo lingüístico. La segunda, la sintáctica, compete a la gramaticalización de un subordinante. En nuestro análisis, la tendencia de gramaticalización de este elemento mostró dos directrices, aunque en ambas se involucra un 
elemento deíctico, demostrativo o pronominal. En la primera de ellas, el subordinante, al ocurrir en construcciones relativas, atrae a demostrativos; esto se observó en tarahumara en el subordinante mapu; en tepehuano del norte en los subordinante que atraen elementos interrogativos, iš=máákidi y ši=anki; y en tepehuano del sur en el subordinante complejo al que se le adjuntan clíticos pronominales de persona. En todas estas lenguas se observó que de alguna manera un subordinante confluye, bien con un demostrativo, o bien, con un clítico pronominal, para situarse justo al inicio de la construcción relativa. La gramaticalización más extrema de esta cadena se manifiesta en tarahumara, donde el nexo mapuse ha fusionado a un antiguo demostrativo pu, cuya vitalidad hoy ya se ha perdido. La segunda faceta de la gramaticalización de un demostrativo es la del pima bajo (Estrada Fernández 2012). En este caso, la gramaticalización no parte de un subordinante, sino de un sufijo nominalizador estativo al que se le une un demostrativo. Estas tres rutas de cambio se resumen en la Tabla 5.

\begin{tabular}{|llll|}
\hline Morfológicas & Sintácticas & & \\
& I & II & III \\
Yaqui & Nmlz. & Sub. + Dem. & Nmlz. + Dem. \\
Guarijio & $-m e /-$ 'u & --- & --- \\
Ópata & - me & --- & --- \\
Tarahumara & $-k a,-k a m e$ & --- & --- \\
Tepehuano del norte & $-a,-a m e$ & mapu & --- \\
Tepehuano del sur & - -kame & ši $\sim$ iš=INTER & --- \\
Névome & - came & na=CL.PRON & --- \\
Pima Bajo & --- & --- & - cugai \\
& & -- & - kig \\
\hline
\end{tabular}

Tabla 5. Cadenas de gramaticalización en construcciones relativas

Dar una explicación de la tendencia de cambio que da origen a un relativizador del tipo que solamente se manifiesta en névome y pima bajo moderno obliga a plantear las siguientes tres interrogantes: ¿Es esta ruta de gramaticalización heredada de otras lenguas yuto-aztecas?, ¿es una ruta de gramaticalización cuya manifestación debe investigarse en otras lenguas yutoaztecas más distantes?, o bien, ¿deben explorarse sus orígenes en una zona geográfica más amplia considerando lenguas de otras familias lingüísticas? La explicación se hace nítida al atender precisamente el aspecto areal.

En un estudio sobre construcciones relativas del coahuilteco (lengua extinta de Texas y no genéticamente relacionada con las yuto-aztecas), Troike (2010: 111) observa que la lengua mostraba un demostrativo al final del nombre relativizado (N-RC-Dem), estrategia que, según el autor, es "apparently unique in North America"; lo cual se confirma al observar el ejemplo en (26) donde un demostrativo ocurre al final del verbo de una construcción relativa: 
26. Coahuilteco (Troike, 2010: 118$)^{25}$

$\begin{array}{lll}\text { pin (xami-n) e } \quad \text { xa-p-o-wxčalak } & \text { pitapa-m. } \\ \text { thing (you) OBJ } 2>3-\text {-sUB-steal } & \text { DEM-2C } \\ \text { 'that thing (which) (you) stole.' } & \end{array}$

Otra evidencia procede de una lengua no yuto-azteca hablada en una zona no tan distante, el seri, como se observa en el ejemplo (27):

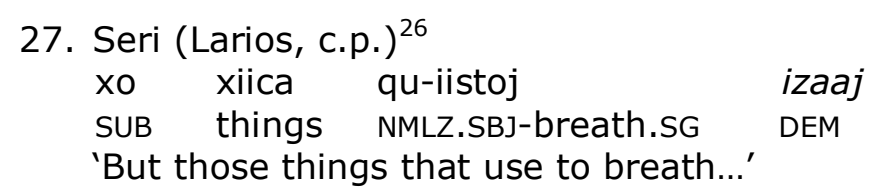

Ninguna de las dos lenguas, ni el seri ni el coahuilteco, guardan una relación genética con las yuto-aztecas; sin embargo, coinciden en que ambas se encuentran en un área lingüística geográfica -la zona norte de México, sur de los Estados Unidos- en la que también habitaban hablantes de las lenguas yuto-aztecas que hemos descrito. Un factor relevante de los pueblos hablantes de estas lenguas fue su carácter nómada regido sobre todo por los veranos e inviernos de temperatura extrema. Dar una explicación contundente al surgimiento de esta estrategia única en las lenguas yuto-aztecas del noroeste de México, requiere incursionar en la búsqueda de argumentos extralingüísticos para los que seguramente no podremos contar con evidencias lo suficientemente sólidas para su demostración. La prehistoria antropológica y lingüística de los pueblos nómadas del sur de los Estados Unidos y el norte de México permanece abierta a la investigación. Sin embargo, las evidencias internas, provenientes de la comparación lingüística que nos proporciona el ejemplo en (27) favorece, sin lugar a dudas, el argumento a favor del contexto lingüístico que seguramente influyó en el patrón observado en pima bajo y el antiguo névome.

\section{Conclusiones}

El objetivo central de esta contribución fue el reflexionar sobre las rutas de gramaticalización de ciertos fenómenos de cambio en lenguas yuto-aztecas del noroeste de México. El análisis abordó tres tipos de construcciones, un clasificador de posesión animal, verbos que se insertan en el llamado dominio de la voz media y las estrategias de relativización. El análisis comparativo de los fenómenos, antes mencionados, nos permitió mostrar un escenario diverso sobre las posibles rutas de gramaticalización que se observan en lenguas de una misma familia y que además comparten límites geográficos colindantes. La investigación intratipológica, con lenguas de la misma familia nos permitió tener claras las expectativas de cambio que se presentan en las diversas lenguas discutidas, dígase la gramaticalización heredada. Sin embargo, los datos también abren la puerta a la posibilidad de discutir aspectos de la gramaticalización inducida por el contacto, sea por situaciones de bilingüismo,

\footnotetext{
${ }^{25}$ Los índices referenciales marcados por el autor se han omitido.

${ }^{26}$ Notas de campo de Maria Alfonsa Larios.
} 
como el que sucede ante el español, que ha afectado a estas lenguas en los últimos cuatrocientos años, o bien, la de una zona areal de mayor amplitud que se tendría que explorar más sistemáticamente, pero que hasta ahora nos proporciona evidencias que no podemos descartar para poder prever la existencia de un área lingüística prehispánica en la zona del norte de México y el sur de los Estados Unidos.

\section{Abreviaturas}

1, 2, 3: primera, segunda o tercera persona, ACU: acusativo, AF: afirmativo, ANT: anterior, APL: aplicativo, ASP: aspecto, B: baseauxiliar, CIT: citativo, CoMP: completivo, COND: condicional, CONJ: conjunción, CONT: continuo, DAT: dativo, DEM: demostrativo, DET: determinante, DIM: diminutivo, DIR: direccional, DPD: delimitador prosódicodiscursivo, DUR: durativo, DV: deverbalizador, E: iqui, EST: estativo, EVI: evidencial, FOC: focus, FUT: futuro, GEN: genitivo, HAB: habitual, IMP: imperativo, IMPF: imperfectivo, INAL: inalienable, INT: intensificador, INTER: interrogativo, IRR: irrealis, IT: iterativo, LOC: locativo, MIR: mirativo, NEG: negativo, NOM: nominativo, NMLZ: nominalizador, NSUJ: no-sujeto, OBJ: objeto, OBL: oblicuo, PFV: perfectivo, PL: plural, POS: posesivo, PRS: presente, PRTC: participio, PASD: pasado, RDP: reduplicación, REL: relativizador, REM: remoto, REP.ID: reportativo.información desconocida, RFLX: reflexivo, SBJ: subject, SUJ: sujeto, SG: singular, SUB: subordinante.

\section{Referencias}

Bahr, Donald M., Juan Gregorio, David I. Lopez y Albert Alvarez. 1974. Piman Shamanism and Staying sickness (Ká:cim Múmkidag), Tucson, The University of Arizona Press.

Bascom, Burt. 1982. Northern Tepehuan, en Ronald W. Langacker (ed.), Studies in Uto-Aztecan grammar. Vol. 3. Uto-Aztecan grammatical sketches, Dallas, Summer Institute of Linguistics/University of Texas at Arlington: 267-393.

Bisang, Walter. 2015. Problems with primary vs. secondary grammaticalization: the case of East and mainland Southeast Asian languages, Language Sciences, 47: 132-147.

Brambila, David. 1953. Gramática rarámuri, México, Editorial Buena Prensa.

Estrada Fernández, Zarina. 2005. The pronominal form a- in Pima Bajo as a middle marker, en International Journal of American Linguistics, 71, 3: 277-302.

Estrada Fernández, Zarina. 2006. Caracterización de la voz media en pima bajo, en Lingüística Mexicana, 3, 1: 5-22.

Estrada Fernández, Zarina. 2012. From demonstrative to a relative marker to clause linker: The relative clause formation in Pima Bajo, en Bernard Comrie y Zarina Estrada-Fernández (eds.), Relative clauses in languages of the Americas: a typological overview. Amsterdam, John Benjamins: 127-146. 
Estrada Fernández, Zarina y Rolando Félix Armendáriz. 2010. Typological differences among middle constructions in some Uto-Aztecan languages, en Claudine Chamoreau, Zarina Estrada Fernández y Yolanda Lastra (eds.), A new look at language contact in Amerindian languages, München, Lincom-EUROPA: 89-118.

Félix Armendáriz, Rolando. 2007. A grammar of River Warihío, München, LINCOM-Europa.

García Salido, Gabriela. 2007. La voz media en tepehuano del sur, en Zarina Estrada-Fernández et al. (eds.), Mecanismos de voz y formación de palabra, México, Plaza y Valdés: 156-173.

García Salido, Gabriela. 2014. Clause linkage in Southeastern Tepehuan, a Uto-Aztecan Language of Northern Mexico. Ph. D. Dissertation. Universidad de Texas en Austin. Disponible en https://repositories.lib.utexas.edu/handle/2152/27139

García Salido, Gabriela y Zarina Estrada Fernández. 2009. La voz reflexiva, recíproca y media: el caso del tepehuano del sur, en Susana Cuevas (ed.), Lengua y la Antropología por un conocimiento global del hombre. Homenaje a Leonardo Manrique, México, INAH: 253-266.

Givón, Talmy. 1971. Historical syntax and synchronic morphology: an archaeologist's field trip, en Papers from the Regional Meetings of the Chicago Linguistic Society, 7: 394- 415.

Givón, T. 1991. The evolution of dependent clause morpho-syntax in Biblical Hebrew, en Elizabeth C. Traugott y Bernd Heine (eds.), Grammaticalization, vol. 2: Types of grammatical markers, Amsterdam, John Benjamins: 257-310.

Givón, T. 2001. Syntax. An introduction, Vols. 1 y 2, Amsterdam, John Benjamins.

Givón, T. 2015. Diachrony of grammar, Vols. 1 y 2, Amsterdam, John Benjamins.

Givón, T. 2016. Nominalization and re-finitization, en Claudine Chamoreau y Zarina Estrada-Fernández. Finiteness and Nominalization, Amsterdam, John Benjamins: 271-296.

Guadalaxara, Thomas de, S. J. 1683. Compendio del arte de la lengua de los tarahumares, y guazápares, Puebla de los Ángeles, Diego Fernández de León.

Guerrero, Lilián. 2012. On relative clauses and related constructions in Yaqui, en Bernard Comrie y Zarina Estrada Fernández (eds.), Relative clauses in languages of the Americas: a typological overview, Amsterdam, John Benjamins: $97-126$.

Haspelmath, Martin. 1993. More on the typology of inchoative-causative verb alternation, en Bernard Comrie y Maria Polinsky (eds.), Causatives and transitivity, Amsterdam, John Benjamins: 87-120.

Heine, Bernd, Ulrike Claudi y Friederike Hünnemeyer. 1991. Grammatizalization. A conceptual framework, Chicago y London: The Chicago University Press.

Heine, Bernd y Tania Kuteva. 2002. Word lexicon of grammaticalization, Cambridge, Cambridge University Press. 
Heine, Berndy y Tania Kuteva. 2007. Genesis of grammar, Oxford, Oxford University Press.

Kemmer, Suzanne. 1993. The middle voice,Amsterdam, John Benjamins.

Kemmer, Suzanne. 1994. Middle voice, transitivity and events, en Barbara Fox y Paul J. Hopper (eds.), Voice form and function, Amsterdam, John Benjamins: $179-230$.

Klaiman, M. H. 1992. Middle verbs, reflexive middle constructions and middle voice, Studies in Language, 16, 1: 35-61.

Kuryłowicz, Jerzy. 1965. L'évolution des catégories gramaticales, Diogenes, 51: 54-71.

Lehmann, Christian. 2002. Thoughts on grammaticalization. Disponible en http://www.christianlehmann.eu/publ/ASSidUE09.pdf Consultado el 201412-16.

Manney, Linda Joyce. 2000. Middle voice in Modern Greek: Meaning and function of an inflectional category, Amsterdam, John Benjamins.

Malchukov, Andrej L. 2004. Nominalization/verbalization: constraining typology of transcategorial operations, München, Lincom Europa.

Meillet, Antoine. 1912. L'évolution des formes gramaticales, en Scientia (Rivista internazionale di sintesi scientifica), 12, 26: 384-400.

Mel'čuk, Igor. 1993. The inflectional category of voice: towards a more rigorous definition, en Bernard Comrie y Maria Polinsky (eds.), Causatives and transitivity, Amsterdam, John Benjamins: 1-46.

Miller, Wick R. 1980. The Possession of pets, dogs, and other beasts (both animal and human) in Southern California, and surrounding areas, $A A A$ Annual Meeting, Washington, D.C. Disponible en https://collections.lib.utah.edu/details?id =362512

Miller, Wick R. 1996. Guarijío: Gramática, textos y vocabulario, México, Universidad Nacional Autónoma de México.

Mithun, Marianne. 2012. Questionable relatives, en Bernard Comrie y Zarina Estrada Fernández (eds.), Relative clauses in languages of the Americas: a typological overview, Amsterdam, John Benjamins: 269-300.

Peralta Ramírez, Valentín. 2015. Cambios morfosintácticos en el náhuatl general. El paso de una lengua yuto-nahua norteña a una lengua mesoamericana, en Zarina Estrada Fernández, Ana Fernández Garay y Albert Alvarez Gonzalez (eds.), Estudios de lenguas amerindias 3: Escenarios de diversidad lingüística, Hermosillo, Universidad de Sonora: 127-168.

Ramírez Mendívil, Moisés David. Inédito. La lengua ópata: entre la nominalización y la finitud. Tesis de Doctorado en Humanidades. Hermosillo, Universidad de Sonora.

Shaul, David L. 1986. Topics in Nevome syntax, Berkeley, University of California Press.

Shibatani, Masayoshi. 2007. Grammaticalization of converb constructions. The case of Japanese -te conjunctive constructions, en Johen Rehbein, Christiane Hohenstein y Lukas Pietsch (eds.), Connectivity in Grammar and Discourse, Amsterdam, John Benjamins: 21-49.

Steffel, Matthäus. Ms. 1799. Gramaticae linguae tarahumaricae, Archiv Mesta Brna, file v3, Knihova Mittrovsky, Sign. A62. 
Troike, Rudolph. C. 2010. The typology of relative clauses in Coahuilteco, an Indian language of Texas, Southwest Journal of Linguistics, 29, 1: 111132.

Wiemer, Björn. 2014. Quo vadis grammaticalization theory? Why complex language change is like words. Folia Linguistica, 48, 2: 425-467.

Willett, Thomas L. 1991. A reference grammar of Southeastern Tepehuan, Dallas, The Summer Institute of Linguistics/ The University of Texas at Arlington. 